Title : will be set by the publisher

Editors : will be set by the publisher

EAS Publications Series, Vol. ?, 2018

\title{
TWO-DIMENSIONAL MODELS OF EARLY-TYPE FAST ROTATING STARS: NEW CHALLENGES IN STELLAR PHYSICS
}

\author{
Michel Rieutord and Francisco Espinosa Lara ${ }^{1}$
}

\begin{abstract}
Two-dimensional models of rapidly rotating stars are already unavoidable for the interpretation of interferometric or asteroseismic data of this kind of stars. When combined with time evolution, they will allow the including of a more accurate physics for the computation of element transport and the determination of surface abundances. In addition, modeling the evolution of rotation will improve gyrochronology.

Presently, two-dimensional ESTER models predict the structure and the large-scale flows (differential rotation and meridional circulation) of stars with mass larger than $1.7 \mathrm{M}_{\odot}$ at any rotation rate. Main sequence evolution can be mimicked by varying the hydrogen content of the convective core. Models have been successfully tested on half a dozen of nearby fast rotating stars observed with optical or infra-red interferometers. They are now the right tool to investigate the oscillation spectrum of early-type fast rotators.
\end{abstract}

\section{Introduction}

One of the main challenges of nowadays stellar physics is the building of twodimensional stellar models including mean flows that pervade the stars. These flows might have various origins but rotation is certainly the most common.

It has long been known that rotation breaks the spherical symmetry of a star but the contained angular momentum has also many consequences on the internal dynamics of such stars. The Coriolis acceleration for instance results from the conservation of angular momentum by fluid parcels. It imposes a complicated dynamics with many types of shear layers (Ekman or Stewartson layers) and allow new wave systems (inertial waves). Radiative zones cannot be at rest as they are submitted to the baroclinic torque. The resulting baroclinic flows together

${ }^{1}$ Université de Toulouse; UPS-OMP; IRAP; Toulouse, France and CNRS; IRAP; 14, avenue Edouard Belin, F-31400 Toulouse, France 
Title : will be set by the publisher

with spin-down or spin-up flows coming from mass-loss or mass contraction are at the origin of the so-called rotational mixing. Rotational mixing designates the effects of both the meridional circulation and the small-scale turbulence induced by differential rotation. Much work has been devoted to its insertion in onedimensional codes (see Maeder \& Meynet 2000, for a review). Although undeniable successes have been obtained with such an approach, many questions are still pending when data are examined in closer details. One of them is the limit of validity of $1 \mathrm{D}$ models when fast rotation has to be considered.

In this contribution, we would like to show that the future of stellar modeling is in multi-dimensional models and, especially, in two-dimensional models. We show below that two-dimensional models are now realistic enough to help for the interpretation of observational data and to offer new insights in the internal dynamics of rotating stars. A discussion of the future steps in the improvements of such models ends this work.

\section{Why two-dimensional models?}

We may wonder why we should direct the modelling of stars towards 2D (or 3D) models and not try to still improve 1D models that have proved so effective in the past. The main reason comes from the strengthening necessity of accounting for large-scale fluid flows in stars so as to better understand the effects of secular transport on chemical abundances. Except for a radial expansion or contraction, fluid flows need at least two-dimensions of space to ensure mass-conservation in a meridional motion. Indeed, using spheroidal coordinates, a steady flow requires that

$$
\partial_{\zeta}\left(\sqrt{|g|} \rho V^{\zeta}\right)+\partial_{\theta}\left(\sqrt{|g|} \rho V^{\theta}\right)=0
$$

with obvious notations (see Rieutord \& Espinosa Lara 2013). The meridional components of the velocity field can therefore be expressed with a stream function $\psi$ such that

$$
V^{\zeta}=\left(\partial_{\theta} \psi\right) / \rho \sqrt{|g|}, \quad V^{\theta}=-\left(\partial_{\zeta} \psi\right) / \rho \sqrt{|g|}
$$

It shows that vertical transport (by $V^{\zeta}$ ) requires latitudinal variations of the stream function. Hence, 2D models are the minimum step to include fluid flows in a somewhat realistic way.

However, beyond this a priori theoretical requirement, observational data also force us to forget about the spherical symmetry. Let us discuss the main points.

\subsection{Images of fast rotators}

Since the work of Domiciano de Souza et al. (2003) on Achernar (see Fig. 1), imaging the surface of nearby rotating stars with optical or IR interferometers has made substantial progresses (e.g. Monnier et al. 2007, and Fig. 22). Presently, the shape and the basic brightness distribution is measured for a ten of stars. But the 
derivation of fundamental parameters of these stars relies on models that are used to match interferometric visibilities or closure phases. So the better the models, the better the parameters. Up to now (e.g. Monnier et al. 2012), Roche models combined with von Zeipel laws of gravity darkening are used to invert these data. These models are very basic and more realistic ones are extremely welcome to give more accurate measurements.

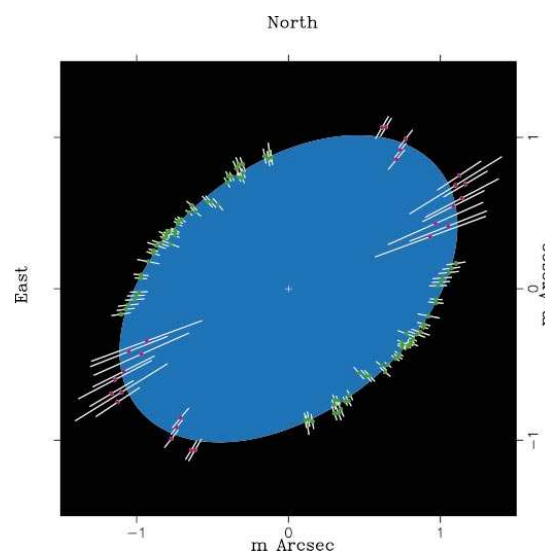

Fig. 1. First determination of Achernar's shape with VLTI (Domiciano de Souza et al. 2003).

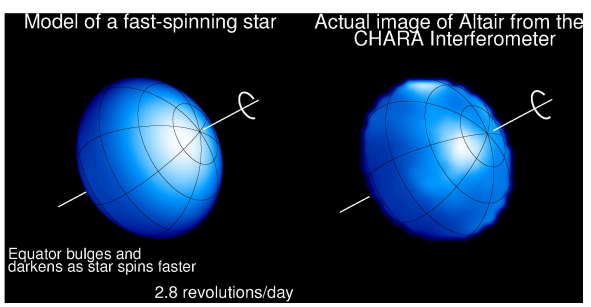

Fig. 2. Reconstruction of the surface brightness of Altair with CHARA interferometer by Monnier et al. (2007).

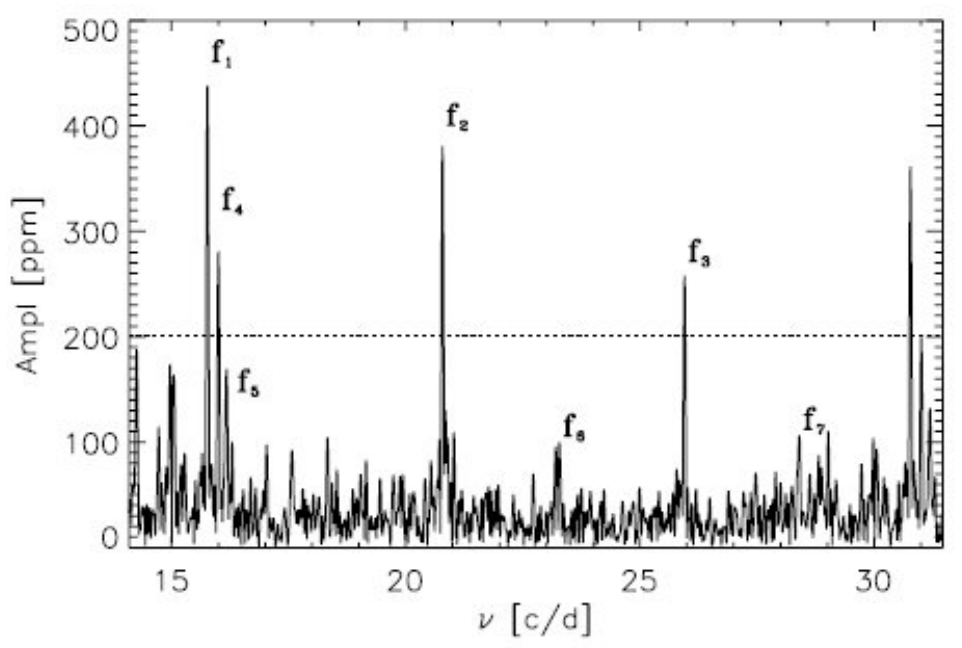

Fig. 3. Part of the oscillation spectrum of Altair from WIRE (Buzasi et al. 2005). 
Title : will be set by the publisher

\subsection{Asteroseismology}

The large amount of photometric data that have been collected by space missions like CoRoT, KEPLER, MOST or WIRE include a large number of rapidly rotating stars. These data, like those shown in Fig. 3 are waiting for an interpretation. This interpretation is of crucial importance to get a direct view on the interior of early-type stars. Apart a few exception, all these stars rotate rapidly. They are powered by the $\mathrm{CNO}$ cycle in a convective core. A long-standing question about them is the size of this core and the amount of overshooting of convection in the envelope. As shown by first investigations (e.g. Rieutord 2006; Espinosa Lara \& Rieutord 2013), the core-envelope interface is likely a complicated region from the dynamical point of view. Asteroseismic constraints are therefore most welcome to select the right models. Since fast rotation is an unavoidable feature of these stars, 2D models are necessary to identify, interpret and invert the associated asteroseismic data. The CoRoT target HD43317, a main sequence B-type star rotating at $50 \%$ of its critical velocity, is certainly the best illustration of this need (see Pápics et al. 2012).

\subsection{Abundance patterns}

1D-models have long been used to investigate the surface abundances in relation with rotational mixing (e.g. Maeder 2009) with true success. However, recent works based on large spectroscopic surveys with the VLT, comparing observed abundance of nitrogen and prediction of models with the so-called Hunter diagram (Brott et al. 2011) show obvious discrepancies between models an observations. The long-standing questions about the abundances of lithium or beryllium are still stimulating research on the way these elements are transported and depleted (e.g. Tognelli et al. 2012). Clearly, more refined models that describe mixing associated with rotation are needed.

\subsection{Other fundamental questions about rotating stars}

Even if observations prompt us to difficult questions (like the previous ones), the conception of models or simply our curiosity about the evolution of stars, rises

other fundamental questions on the role of rotation in stellar evolution. A few of them are the following:

- What is the dynamical evolution of rotating PMS stars? In which dynamical state do they reach the main sequence?

- First stars, so-called population III stars, were lacking metals and therefore were more compact, concentrating angular momentum. What has been the evolution and the yields of these stars whose rotation has been certainly quite fast?

- It is conjectured that rotation plays a crucial role in the explosion of supernovae that produce gamma ray burst. What is the pre-supernova dynamical 
state?

\subsection{Conclusions}

All the foregoing arguments show that two-dimensional models including rotation at any rate will permit the detailed exploration of numerous very timely problems. It is one of the grand challenges of stellar physics for the next decades.

\section{Constructing 2D models}

\subsection{Basic assumptions}

In order to make the problem well-posed and tractable, we consider an isolated, non-magnetic rotating star. Presently, we discard any mass-loss or time-evolution and search for steady state solutions. We thus look for the structure of such stars including their large-scale flows. Such models may not be very far from actual early-type stars (typically A and B stars).

\subsection{Equations to be solved}

Let us just recall that the foregoing problem is that of the flow of a self-gravitating compressible plasma. It is controlled by the four following partial differential equations:

$$
\left\{\begin{array}{l}
\Delta \phi=4 \pi G \rho \\
\rho T \vec{v} \cdot \vec{\nabla} S=-\operatorname{div} \vec{F}+\varepsilon_{*} \\
\rho\left(2 \vec{\Omega}_{*} \wedge \vec{v}+\vec{v} \cdot \vec{\nabla} \vec{v}\right)=-\vec{\nabla} P-\rho \vec{\nabla}\left(\phi-\frac{1}{2} \Omega_{*}^{2} s^{2}\right)+\vec{F}_{v} \\
\operatorname{div}(\rho \vec{v})=0 .
\end{array}\right.
$$

namely Poisson equation, entropy equation, momentum equation and mass conservation equation respectively. These equations are completed by the microphysics prescriptions

$$
\left\{\begin{array}{l}
P \equiv P(\rho, T) \quad \text { OPAL } \\
\chi_{r} \equiv \chi_{r}(\rho, T) \quad \text { OPAL } \\
\varepsilon_{*} \equiv \varepsilon_{*}(\rho, T) \quad \text { NACRE }
\end{array}\right.
$$

and some transport prescriptions:

- The energy flux

$$
\vec{F}=-\chi_{r} \vec{\nabla} T-\frac{\chi_{\text {turb }} T}{\mathcal{R}_{M}} \vec{\nabla} S
$$

- The transport of momentum

$$
\begin{aligned}
\vec{F}_{v}=\mu \overrightarrow{\mathcal{F}}_{\mu}(\vec{v})=\mu & {\left[\Delta \vec{v}+\frac{1}{3} \vec{\nabla}(\vec{\nabla} \cdot \vec{v})+2(\vec{\nabla} \ln \mu \cdot \vec{\nabla}) \vec{v}\right.} \\
& \left.+\vec{\nabla} \ln \mu \times(\vec{\nabla} \times \vec{v})-\frac{2}{3}(\vec{\nabla} \cdot \vec{v}) \vec{\nabla} \ln \mu\right] .
\end{aligned}
$$


or any mean-field expression of the Reynolds stress.

Boundary conditions read

- On pressure (radiative equilibrium)

$$
P_{s}=\frac{2}{3} \frac{\bar{g}}{\bar{\kappa}}
$$

- On the velocity field (stress-free conditions)

$$
\vec{v} \cdot \vec{n}=0 \quad \text { and } \quad([\sigma] \vec{n}) \wedge \vec{n}=\overrightarrow{0}
$$

- On temperature (black body radiation)

$$
\vec{n} \cdot \vec{\nabla} T+T / L_{T}=0
$$

with a prescription of the angular momentum content or of the equatorial velocity, namely:

$$
\int_{(V)} r \sin \theta \rho u_{\varphi} d V=L \quad \text { or } \quad v_{\varphi}(r=R, \theta=\pi / 2)=V_{\mathrm{Eq}}
$$

Detailed comments may be found in Espinosa Lara \& Rieutord (2013).

\section{The ESTER project and some results}

\subsection{Present status}

We may briefly present the ESTER code as follows:

- It is a spectral code (Chebyshev polynomials \& spherical harmonics) written in $\mathrm{C}++\&$ Python. It is an open source code (http://code.google.com/p/esterproject).

- The spheroidal shape of the star is accounted through a mapping of the natural coordinates towards spherical ones (see Rieutord \& Espinosa Lara 2013).

- It solves the structure equations in the asymptotic limit of small viscosities with the Newton algorithm.

- It has been tested through comparisons with $1 \mathrm{D}$ codes at $\Omega=0$.

- Solutions are checked with internal tests (virial, energy integral, spectral convergence, roundoff errors). 

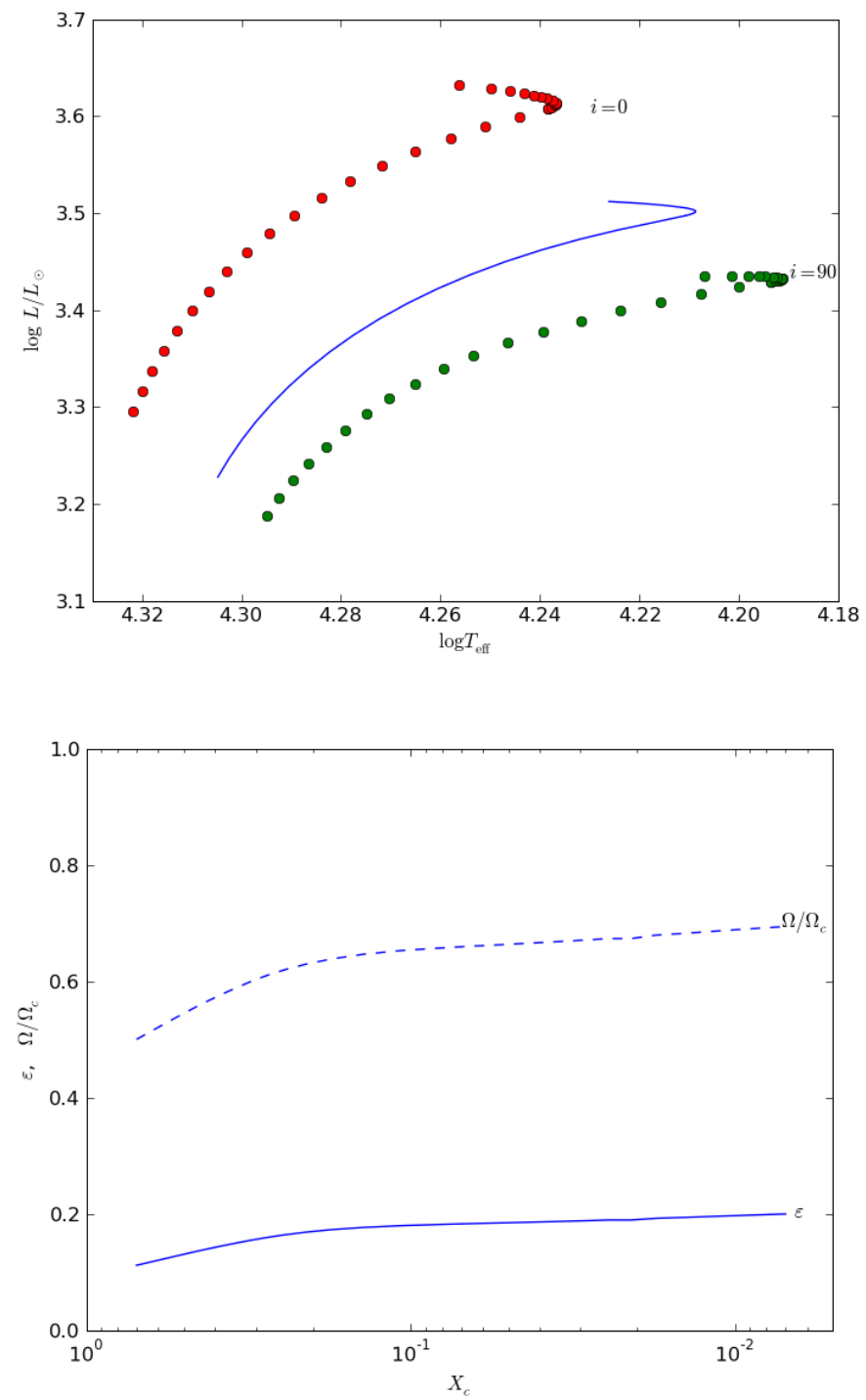

Fig. 4. Variation of the position of a $7 \mathrm{M}_{\odot}$ star in the HR diagram when the hydrogen content of its core is decreased. The apparent trajectory that depends on the inclination $i$ of the rotation axis with respect to the line of sight is given for $i=0$ (pole on) and $i=90^{\circ}$ (equator on).

Presently, the ESTER code can compute models for early-type stars of mass larger than $1.7 \mathrm{M}_{\odot}$ rotating up to breakup. In Tab. 11, we give the example of 
Title : will be set by the publisher

Table 1. Comparison between observationally derived parameters of the stars and tentative two-dimensional models. Data from Altair are from Monnier et al. (2007) and those of Alderamin are from Zhao et al. (2009). Here $\varepsilon$ is the flatness, $\omega_{k}$ the ratio of the equatorial angular velocity to the keplerian one, $j$ is the mean specific angular momentum of the star and $\mathrm{X}_{\mathrm{env}}$. is the hydrogen mass fraction in the envelope.

\begin{tabular}{lllll}
\hline Star & \multicolumn{2}{c}{ Altair $(\alpha$ Aql $)$} & \multicolumn{2}{c}{ Alderamin $(\alpha$ Cep $)$} \\
& Observations & Model & Observations & Model \\
& & & & \\
Spectral type & A7 IV-V & & A8 V & \\
Mass $\left(\mathrm{M}_{\odot}\right)$ & & 1.65 & $1.92 \pm 0.04$ & 1.92 \\
$\mathrm{R}_{\text {eq }}\left(\mathrm{R}_{\odot}\right)$ & $2.029 \pm 0.007$ & 2.027 & $2.739 \pm 0.04$ & 2.747 \\
$\mathrm{R}_{\text {pol }}\left(\mathrm{R}_{\odot}\right)$ & $1.634 \pm 0.011$ & 1.627 & $2.199 \pm 0.035$ & 2.166 \\
$\varepsilon$ & & 0.197 & & 0.211 \\
$\omega_{k}$ & & 0.695 & & 0.725 \\
$i$ & $57^{\circ}$ & & $56^{\circ}$ & \\
$\mathrm{T}_{\text {eq }}(\mathrm{K})$ & $6860 \pm 150$ & 6849 & $6574 \pm 200$ & 6781 \\
$\mathrm{~T}_{\text {pol }}(\mathrm{K})$ & $8450 \pm 140$ & 8450 & $8588 \pm 300$ & 8515 \\
$\mathrm{~L}\left(\mathrm{~L}_{\odot}\right)$ & & 10.2 & $18.1 \pm 1.8$ & 18.6 \\
$\mathrm{~V}_{\text {eq }}(\mathrm{km} / \mathrm{s})$ & $285.5 \pm 6$ & 274 & $272 \pm 18$ & 265 \\
$\mathrm{j}\left(10^{17} \mathrm{~cm}{ }^{2} / \mathrm{s}\right)$ & & 1.12 & & 1.27 \\
$\mathrm{k}\left[\mathrm{j} / \mathrm{R}_{\text {eq }}^{2} \Omega_{\text {eq }}\right]$ & & 0.0289 & & 0.0251 \\
$\mathrm{P}_{\text {eq }}($ days & & 0.374 & & 0.525 \\
$\mathrm{P}_{\text {pol }}($ days $)$ & & 0.387 & & 0.538 \\
$\mathrm{X}_{\text {env. }}$ & & 0.70 & & 0.70 \\
$\mathrm{X}_{\text {core }} / \mathrm{X}_{\text {env. }}$ & & 0.50 & & 0.30 \\
$\mathrm{Z}$ & & 0.014 & & 0.017 \\
\hline
\end{tabular}

Alderamin ( $\alpha$ Cep) and Altair ( $\alpha$ Aql) showing that our models nicely fit the observed fundamental parameters of these stars as determined by interferometry. This is also the case for other A-type stars like Vega or Rasalhague or the B-star $\alpha$ Leo (see Espinosa Lara \& Rieutord 2013). Three other stars, $\delta$ Vel Aa, Ab and Achernar ( $\alpha$ Eri) have also been investigated in Rieutord \& Espinosa Lara (2013). In these models, there is no time evolution but chemical evolution on the main sequence can be mimicked by changing the hydrogen content of the core. In this way we have explored the evolution along the main sequence for stars of constant angular momentum.

The results of such calculations are shown in Fig. 4. In this case the initial rotation rate is $50 \%$ critical. We note that changing the orientation of the rotation axis may change the apparent luminosity by up to a factor 2 (note that limb darkening is not accounted for in this calculation of the apparent luminosity). This same figure is completed by the "evolution" of the angular velocity with 
respect to critical and of the flatness of the surface. As obvious in this diagram, evolution on the main sequence leads to a more and more critical rotation of the star. This comes from the fact that even if the equatorial velocity diminishes because of angular momentum conservation and the growth of the radius, the critical equatorial velocity decreases even more rapidly, making the star's rotation closer and closer to the critical one.

This result clearly shows that stars born at sufficiently rapid rotation rate (typically $60 \%$ critical) will reach the critical rotation on the main sequence. It supports the idea that the Be phenomenon is the result of the evolution of initially fast rotating B-stars, although observations do not seem to show unambiguously a frequency rise of Be stars at the end of the main sequence (Porter \& Rivinius 2003).

\section{Conclusion}

Presently, the ESTER code can compute rather easily models of isolated intermediate-mass or massive stars at any rotation rate. The models are self-consistent in the sense that they do not depend on an arbitrary differential rotation. Their differential rotation (and associated meridional circulation) are computed as the result of the baroclinic torque existing in the radiative envelope. The comparisons of fundamental parameters of the few nearby fast rotators observed with interferometry with ESTER models are quite satisfactory. These models can be used to invert interferometric visibilities and closure phases as they give more realistic gravity darkening profiles than Roche/von Zeipel models (Espinosa Lara \& Rieutord 2011). The next step in comparing models and data is that of the identification of eigenmodes in a fast rotating star (Mirouh et al. 2013; Reese et al. 2013), which is still a challenge.

The future of 2D stellar models is now to move from steady state models to time evolving ones with either gravitational contraction or nuclear reactions networks. As far as structure is concerned, we also plan to extend the capabilities of the

code to low-mass stars, but this needs the development of an efficient modeling of convective envelopes in two-dimensions.

\section{References}

Brott, I., Evans, C. J., Hunter, I., et al. 2011, A\&A, 530, A116

Buzasi, D. L., Bruntt, H., Bedding, T. R., et al. 2005, ApJ, 619, 1072

Domiciano de Souza, A., Kervella, P., Jankov, S., et al. 2003, A\&A, 407, L47

Espinosa Lara, F. \& Rieutord, M. 2011, A\&A, 533, A43

Espinosa Lara, F. \& Rieutord, M. 2013, A\&A, 552, A35

Maeder, A. 2009, Physics, Formation and Evolution of Rotating stars (Springer) 
Maeder, A. \& Meynet, G. 2000, Ann. Rev. Astron. Astrophys., 38, 143

Mirouh, G., Reese, D., Espinosa Lara, F., Ballot, J., \& Rieutord, M. 2013, in IAU Symposium 301, Vol. 865, Precision Asteroseismology, ed. W. Chaplin, J. Guzik, G. Handler, \& A. Pigulski, 1, astro-ph/

Monnier, J., Che, X., Zhao, M., et al. 2012, ApJ, 761, L3

Monnier, J. D., Zhao, M., Pedretti, E., et al. 2007, Science, 317, 342

Pápics, P. I., Briquet, M., Baglin, A., et al. 2012, A\&A, 542, A55

Porter, J. M. \& Rivinius, T. 2003, Pub. Astron. Soc. Pacific, 115, 1153

Reese, D., Espinosa Lara, F., \& Rieutord, M. 2013, in IAU Symposium 301, Vol. 865, Precision Asteroseismology, ed. W. Chaplin, J. Guzik, G. Handler, \& A. Pigulski, 1, astro-ph/

Rieutord, M. 2006, A\&A, 451, 1025

Rieutord, M. \& Espinosa Lara, F. 2013, in Lecture Notes in Physics, Berlin Springer Verlag, Vol. 865, SeIsmology for studies of stellar Rotation and Convection, ed. M. Goupil, K. Belkacem, C. Neiner, F. Lignières, \& J. J. Green, 49-73, astro-ph/1208.4926

Rieutord, M. \& Espinosa Lara, F. 2013, in EAS Publication series, Vol. 1, Proceedings of the Evry Schatzman School 2012, ed. P. Hennebelle \& C. Charbonnel, 1, astro-ph/1305.0496

Tognelli, E., Degl'Innocenti, S., \& Prada Moroni, P. G. 2012, A\&A, 548, A41

Zhao, M., Monnier, J. D., Pedretti, E., et al. 2009, ApJ, 701, 209 